\title{
There and Back Again: Hippocampus and Retrosplenial Cortex Track Homing Distance during Human Path Integration
}

\author{
Elizabeth R. Chrastil, ${ }^{1,2}$ Katherine R. Sherrill, ${ }^{1,2}$ Michael E. Hasselmo, ${ }^{1}$ and Chantal E. Stern ${ }^{1,2}$ \\ ${ }^{1}$ Department of Psychological and Brain Sciences and Center for Memory and Brain, Boston University, Boston, Massachusetts 02215, and ${ }^{2}$ Athinoula A. \\ Martinos Center for Biomedical Imaging, Massachusetts General Hospital, Charlestown, Massachusetts 02129
}

Path integration, the updating of position and orientation during movement, often involves tracking a home location. Here, we examine processes that could contribute to successful location tracking in humans. In particular, we investigate a homing vector model of path integration, whereby a navigator continuously tracks a trajectory back to the home location. To examine this model, we developed a loop task for fMRI, in which participants viewed movement that circled back to a home location in a sparse virtual environment. In support of a homing vector system, hippocampus, retrosplenial cortex, and parahippocampal cortex were responsive to Euclidean distance from home. These results provide the first evidence of a constantly maintained homing signal in the human brain. In addition, hippocampus, retrosplenial cortex, and parahippocampal cortex, as well as medial prefrontal cortex, were recruited during successful path integration. These findings suggest that dynamic processes recruit hippocampus, retrosplenial cortex, and parahippocampal cortex in support of path integration, including a homing vector system that tracks movement relative to home.

Key words: angular gyrus; fMRI; medial prefrontal cortex; navigation; parahippocampal cortex; precuneus

\section{Significance Statement}

Path integration is the continual updating of position and orientation during navigation. Animal studies have identified place cells and grid cells as important for path integration, but underlying models of path integration in humans have rarely been studied. The results of our novel loop closure task are the first to suggest that a homing vector tracks Euclidean distance from the home location, supported by the hippocampus, retrosplenial cortex, and parahippocampal cortex. These findings suggest a potential homing vector mechanism supporting path integration, which recruits hippocampus and retrosplenial cortex to track movement relative to home. These results provide new avenues for computational and animal models by directing attention to homing vector models of path integration, which differ from current movement-tracking models.

\section{Introduction}

Path integration is the constant updating of the navigator's representation of position and orientation during movement without using landmarks (Mittelstaedt and Mittelstaedt, 1982; Byrne et al., 2007) and often involves tracking a start or home location. A continuous homing vector model could account for this track-

\footnotetext{
Received March 27, 2015; revised 0ct. 7, 2015; accepted 0ct. 16, 2015.

Author contributions: E.R.C., K.R.S., M.E.H., and C.E.S. designed research; E.R.C. and K.R.S. performed research; E.R.C. and K.R.S. analyzed data; E.R.C., K.R.S., M.E.H., and C.E.S. wrote the paper.

This work was supported by Office of Naval Research MURI N000 14-10-1-0936. fMRI scanning was completed at the Athinoula A. Martinos Center for Biomedical Imaging (Charlestown, MA), which receives support from NCRR P41RR14075. We thank Andrew Whiteman, Rachel Nauer, and Allen Chang for their assistance; and Sam Ling and Marc Howard for helpful comments.

The authors declare no competing financial interests.

Correspondence should be addressed to Dr. Elizabeth R. Chrastil, Center for Memory and Brain, 2 Cummington Mall, Boston University, Boston, MA 02215. E-mail: chrastil@bu.edu.

DOI:10.1523/JNEUROSCI.1209-15.2015

Copyright $\odot 2015$ the authors $\quad 0270-6474 / 15 / 3515442-11 \$ 15.00 / 0$
}

ing behavior. According to this model, the navigator maintains a vector back to its home location at all times (Fujita et al., 1990; Philbeck et al., 2001). The navigator does not necessarily encode the trajectory of the traveled path but simply maintains information relating their current position to the home location.

The goal of this study was to test the contributions of the homing vector model to human path integration and to examine the brain regions that support this process. We developed a loop closure task, in which participants viewed movement in a virtual environment and decided whether the traversed path returned to the start location. Loop closure is a novel experimental approach to understanding path integration, which removes potential confounding errors in controlling movement during navigation. Loop closure is also a major challenge in robotics (Leonard and Feder, 2000; Durrant-Whyte and Bailey, 2006), and a greater understanding of human path integration could lend insight into solving this problem. 

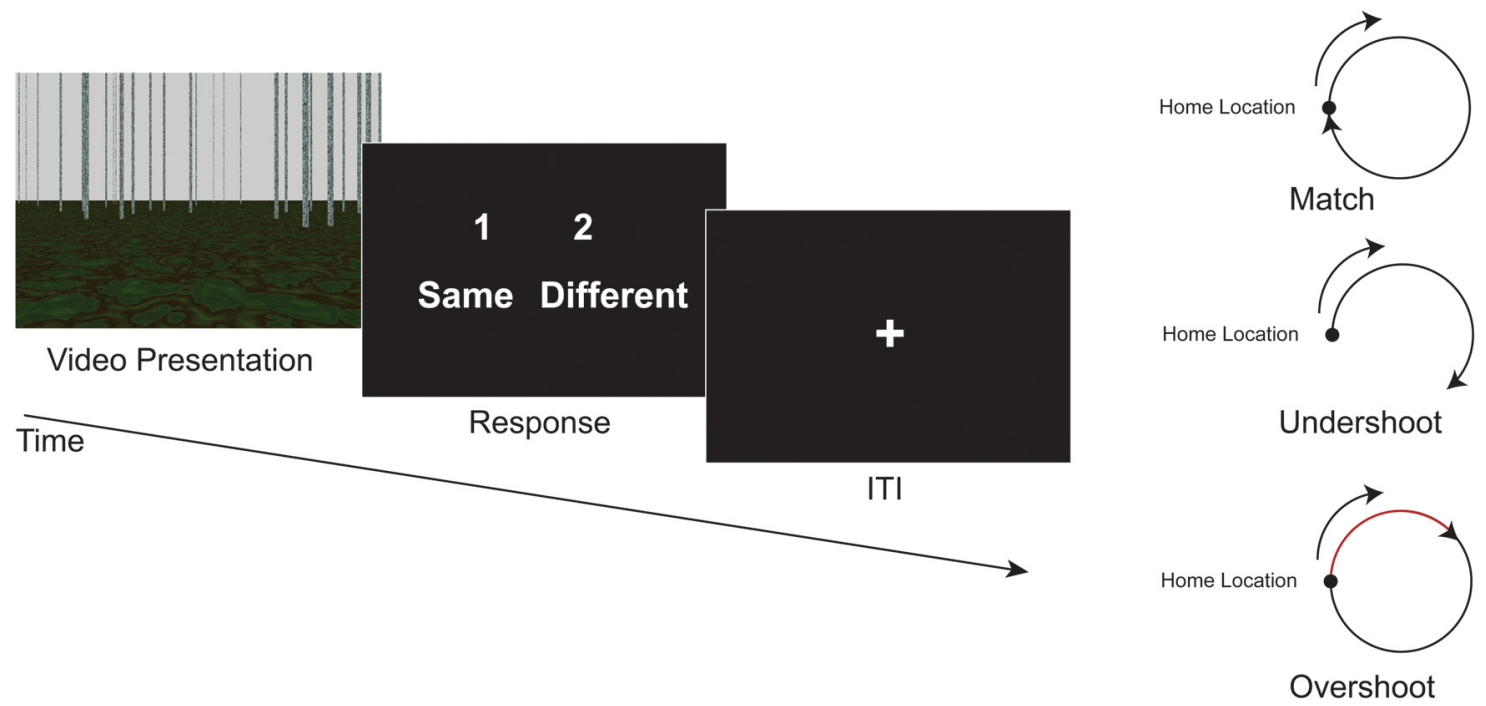

Figure 1. Experimental design. Left, Example view of the environment. Participants first viewed a video of motion along a loop trajectory in a sparse environment. During the response period, they indicated whether the video ended in the same place in which it started ("same," a match trial) or whether it ended in a different location ("different," a mismatch trial). Right, Illustrations of match and mismatch trials. Mismatches could either undershoot the home location or continue past home and go partway around another loop for an overshoot (red).

In rodents, cellular representations that could contribute to path integration include grid cells in entorhinal cortex (Fyhn et al., 2004), place cells in hippocampus (O'Keefe and Nadel, 1978), and head-direction cells in postsubiculum (Taube et al., 1990) and retrosplenial cortex (RSC) (Cho and Sharp, 2001). These signals can be combined to update spatial position during active navigation (Burgess et al., 2007; Hasselmo, 2009; Erdem and Hasselmo, 2012). Lesions in hippocampus and entorhinal cortex have been shown to cause impairments of path integration in rodents (Whishaw et al., 1997; McNaughton et al., 2006; Brun et al., 2008), although some studies have shown minimal path integration impairments with lesions (Alyan and McNaughton, 1999; Kim et al., 2013). RSC has anatomical connections with hippocampus and parahippocampal cortex (PHC) (Sugar et al., 2011; Aggleton et al., 2012), as well as prefrontal cortex (PFC) (Shibata et al., 2004), and lesions to RSC cause impairments in path integration in rodents (Save et al., 2001). Together, these findings suggest roles for hippocampus, $\mathrm{PHC}$, and RSC in processing path integration signals.

Human functional imaging studies demonstrate that several brain regions contribute to path integration. Hippocampal activity predicts accuracy in a path integration task (Wolbers et al., 2007), and in navigation in a sparse environment (Sherrill et al., 2013). In addition, several studies have shown a relationship between hippocampal activation and distance to a goal location (Spiers and Maguire, 2007; Morgan et al., 2011; Viard et al., 2011; Sherrill et al., 2013; Howard et al., 2014). Both hippocampus and PHC are involved in encoding and maintaining translational and rotational information necessary for path integration (E.R.C. et al., under review). Although previous neuroimaging studies have shown that PHC is involved in landmark-based navigation (Janzen and van Turennout, 2004; Epstein and Vass, 2013), PHC activity has also been observed in navigation in landmark-free environments (Sherrill et al., 2013). RSC may play a key role in tracking movement by translating between egocentric (relative to the navigator) and allocentric (independent of the navigator) perspectives (Byrne et al., 2007), and could contribute to processing heading direction (Baumann and Mattingley, 2010; Marchette et al., 2014). Yet, neuroimaging studies have not established the role of these regions in a homing vector model of path integration.

To test the contributions of the hippocampus, $\mathrm{PHC}$, and RSC to location tracking, we developed an fMRI study in which participants tracked their return to a home location during movement along a loop trajectory in a sparse virtual environment. We used a model-based approach to examine changes in fMRI BOLD signal consistent with a homing vector model (Fujita et al., 1990; Philbeck et al., 2001), which tracks the Euclidean (straight-line) distance between individual points on the loop and the home location. Based on theoretical and animal models, our hypothesis was that hippocampus, PHC, and RSC would track Euclidean distance to the home location during navigation, in support of the homing vector model.

\section{Materials and Methods \\ Participants}

Thirty-one participants were recruited for this study from the Boston University community. All participants had no history of neurological disorders. Written informed consent was obtained from each participant before enrollment in accordance with the experimental protocol approved by both the Partners Human Research Committee and the Boston University Charles River Campus Institutional Review Board.

Two participants were eliminated from the final analysis due to excessive motion during fMRI scanning, 3 participants were not scanned due to claustrophobia, 1 participant was found to be ineligible after screening, and 1 participant fell asleep during the scan. Twenty-four participants were included in the final data analysis (mean \pm SD age $23.50 \pm$ 4.81 years; 11 males, 13 females).

\section{Stimuli and tasks}

Environment. The experiment used a virtual environment, developed using POV-Ray version 3.6 (http://www.povray.org/), a 3D ray-tracing modeling program. The environment consisted of a textured ground plane with $\sim 150$ textured poles, or "trees," randomly placed in the scene (Figure 1). The textured ground and trees in the environment provided optic flow information during the video presentation of movement. The trees were taller than the top of the screen so that height changes could not be used as a cue to distance. The large number of trees and random placement discouraged participants from using the scene arrangement as a landmark, and each video had a different random arrangement of the 
trees. Movement in the videos never passed directly through a tree. We emphasize that self-motion information used in this study stems purely from visual motion, with no vestibular or proprioceptive input, due to the constraints of fMRI scanning.

Videos of movement in the environment were presented as a series of images rendered in POV-Ray, at a rate of 30 frames per seconds. The scenes were presented to participants using E-Prime 2.0 (Psychology Software Tools), which also recorded the exact timing of stimulus presentation and participants' responses.

Trial procedure. Each trial began with a loop closure video, which varied in duration (range $\sim 4-25 \mathrm{~s}$, average $11.5 \mathrm{~s}$ ). A response screen followed the loop closure video, and participants had up to $2 \mathrm{~s}$ to respond whether the movement in the loop closure video ended in the home location. A $6 \mathrm{~s}$ intertrial interval (ITI) began immediately when the response was recorded; thus, the duration of the response was indicative of participants' reaction time.

Loop closure. Half of the videos ended in the home location (a full $360^{\circ}$ traversal of the loop; Fig. 1), which we refer to as a "match," and half ended at another point along the circle, which we refer to as a "mismatch." Half of the mismatches were undershoots, such that the movement only traversed a portion of the circle $\left(225^{\circ}\right.$ of the loop). The other half of mismatches were overshoots, such that movement went past the home location and continued partway around a second loop $\left(495^{\circ}\right.$ of the loop). Participants were given clear instructions that undershoots and overshoots were considered mismatches, and that it was important to determine whether the end point itself was the start location. Three different radii of curvature $(2.0,3.0$, and 4.5 virtual units) and two different travel speeds ( 1.5 and 2.0 virtual units/s) were used in the loop closure task to decouple distance, time, and proportion of the circle traveled during the task. Loops turned both to the right and to the left in equal numbers; we combined over left and right turning direction for analysis.

The design of the current study eliminates any potential activation due to motor control or attentional deployment to controlling movement. It also eliminates performance differences due to execution errors in movement. Instead, the attention of the participant is focused on location tracking during path integration.

\section{Procedure}

Prescan training. Participants were trained outside the scanner the day before scanning. Participants were given a general description of movement in the environment and shown a short example video. The practice consisted of 24 trials, and the loops used in the practice, although similar in design, were not the same as those used during scanning. Feedback was provided during the practice; immediately after the response for each trial, participants were presented with information about whether their response was correct and a running average of the percentage correct. In addition to the loop task, participants were trained on 4 other tasks related to path integration (E.R.C. et al., under review). They were then given task-specific instructions and practice for each of the tasks in turn. After one time through all experimental tasks, participants were given the opportunity to take a break and to ask questions about the tasks. Another run through the set of 24 practice trials with feedback was given for the experimental tasks. Finally, participants were given a practice run, similar to how runs would be presented in the scanner: no feedback and each of the five tasks (the loop closure task, discussed here, and the four other path integration tasks) (E.R.C. et al., under review) presented in blocks, with 6 trials per block.

At the conclusion of the prescan training, participants completed several behavioral spatial abilities tasks, which allowed us to examine potential individual differences. These behavioral tasks included the self-report questionnaire Santa Barbara Sense of Direction Scale (Hegarty et al., 2002), a questionnaire about frequency and manner of personal video game use, the Road Map Test in which participants report the direction of each turn in a route predrawn on a city map (Money and Alexander, 1966; Zacks et al., 2000), and the PerspectiveTaking Spatial Orienting Test in which participants view a 2D array of objects on a page and indicate directional relationships from imagined viewpoints (Kozhevnikov and Hegarty, 2001).
Scanning procedure. While structural scans were acquired, participants were given a practice run with feedback using examples from the training session, with 8 trials per task block. Each of the test runs consisted of one block each of the experimental tasks (the loop closure task and the 4 other tasks not presented here). Each block contained 6 trials of the loop closure task, with match and mismatch trials counterbalanced across runs. The task order of each block was counterbalanced across runs. There were six runs, randomized across participants, for a total of 36 trials of loop closure. Because the ITI began as soon as participants made their responses, the scan time for each run varied but generally lasted $<10 \mathrm{~min}$.

\section{Image acquisition}

Images were acquired at the Athinoula A. Martinos Center for Biomedical Imaging, Massachusetts General Hospital in Charlestown, MA using a 3 tesla Siemens MAGNETOM TrioTim scanner with a 32-channel Tim Matrix head coil. A high-resolution T1-weighted MP-RAGE structural scan was acquired using Generalized Autocalibrating Partially Parallel Acquisitions $\left(\mathrm{TR}=2530 \mathrm{~ms} ; \mathrm{TE}=3.31 \mathrm{~ms}\right.$; flip angle $=7^{\circ}$; slices $=176$; resolution $=1 \mathrm{~mm}$ isotropic). $\mathrm{T}^{*}$-weighted $\mathrm{BOLD}$ images were acquired using an EPI sequence $(\mathrm{TR}=2000 \mathrm{~ms} ; \mathrm{TE}=30 \mathrm{~ms}$; flip angle $=$ $85^{\circ}$; slices $=33$, resolution $=3.44 \mathrm{~mm}$ isotropic, interslice gap of 0.5 $\mathrm{mm})$. Functional image slices were aligned parallel to the long axis of the hippocampus. AMRI signal dropout in anterior MTL prevented analysis of entorhinal cortex activity in this study.

\section{fMRI preprocessing}

Functional imaging data were preprocessed and statistically analyzed using the SPM8 software package (Statistical Parametric Mapping, Wellcome Department of Cognitive Neurology, London). All BOLD images were first reoriented so the origin (i.e., coordinate $x, y, z=[0,0,0]$ ) was at the anterior commissure. The images were then corrected for differences in slice timing and were realigned to the first image collected within a series. Motion correction was conducted next and included realigning and unwarping the BOLD images to the first image in the series to correct for image distortions caused by susceptibility $\times$ movement interactions (Andersson et al., 2001). Realignment was estimated using seventh degree B-spline interpolation with no wrapping, whereas unwrap reslicing was done using seventh degree B-spline interpolation with no wrapping. The high-resolution structural image was then coregistered to the mean BOLD image created during motion correction and segmented into white and gray matter images. The bias-corrected structural image and coregistered BOLD images were spatially normalized into standard MNI space using the Diffeomorphic Anatomical Registration using Exponentiated Lie algebra algorithm (Ashburner, 2007) for improved intersubject registration. BOLD images were resampled during normalization to 2 $\mathrm{mm}^{3}$ isotropic voxels and smoothed using a $6 \mathrm{~mm}$ FWHM Gaussian kernel. The normalized structural images of all 24 participants were averaged after normalization for displaying overlays of functional data.

\section{Analysis}

Behavioral performance analysis. Behavioral performance was analyzed using MATLAB (The MathWorks) and SPSS20 (IBM). Within-subjects repeated-measures ANOVAs were used to assess potential differences in accuracy and reaction time between the different sizes of loops and travel speeds.

$f M R I$ analysis. To model the data, separate regressors were created for correct trials and incorrect trials. These served as the two primary regressors. The other experimental tasks, the response period, and ITI were not included in the model. The six motion parameters calculated during motion correction were added to each model as additional covariates of no interest, as were regressors removing signal intensity spikes (using SPM8's ART function developed by Mozes and Whitfield-Gabrieli http://www.nitrc.org/projects/artifact_detect/).

Both whole-brain and a priori ROI analyses were performed. Based on human and animal literature, we had strong a priori hypotheses that the hippocampus contributes to path integration by supporting a homing vector model (Wolbers et al., 2007; Morgan et al., 2011; Sherrill et al., 2013; Howard et al., 2014), that the PHC contributes to path integration 
A

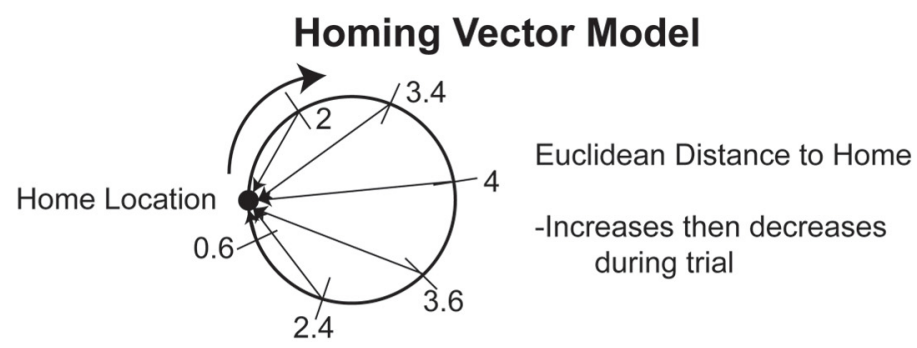

\section{B}
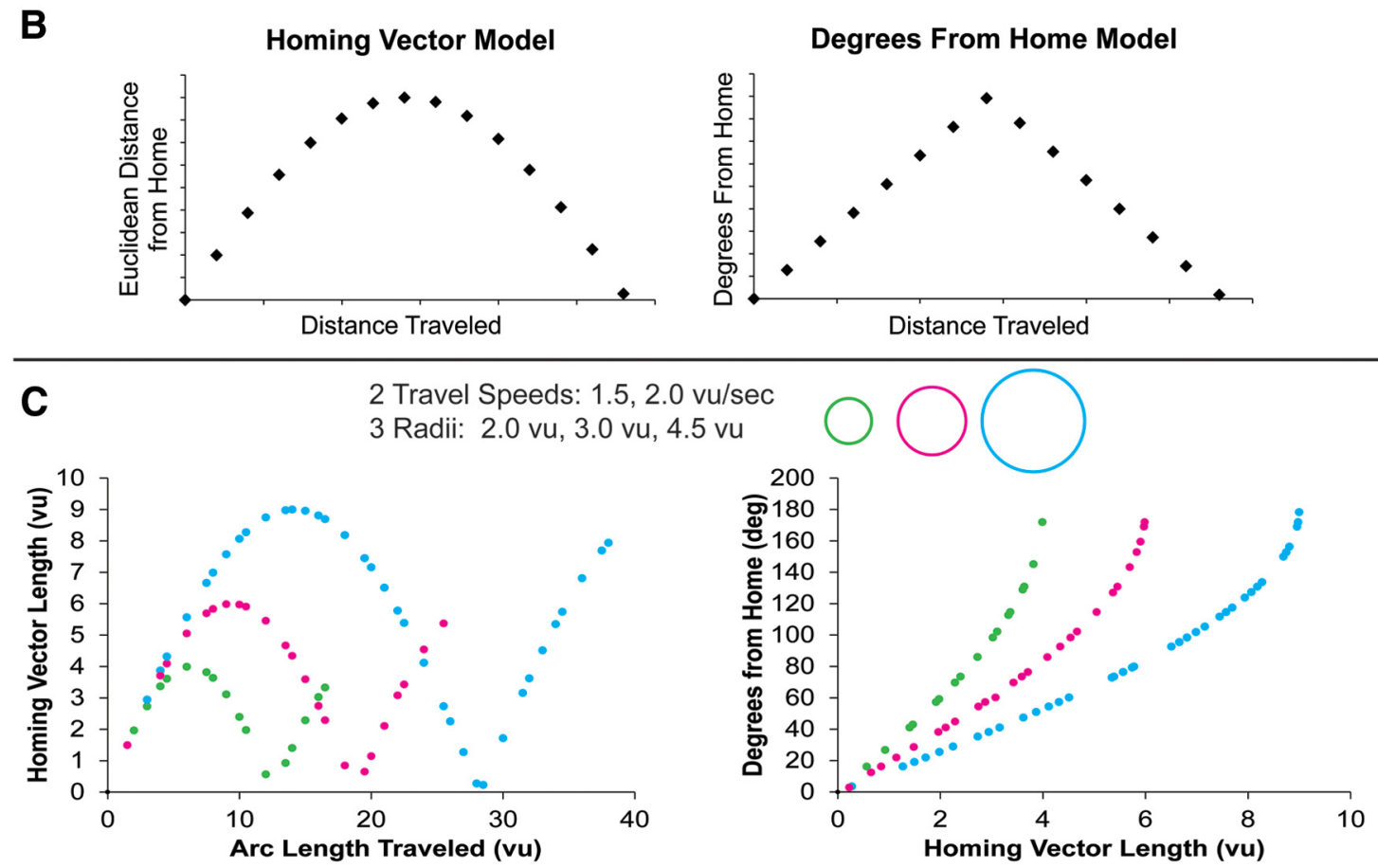

Figure 2. Design of the parametric analysis. $A$, Sticks (onsets with 0 duration) were placed at each second during the loop task, sampling over the entire course of the trial. In this example, the loop is $6.25 \mathrm{slong}$. The homing vector model tracked the length of the chord of the loop between the current location and the home location, equivalent to the Euclidean distance to home. We refer to this length as the homing vector. $\boldsymbol{B}$, Comparison of the Euclidean distance homing vector model and the degrees-from-home model in a single trial example. $C$, We used three loop sizes (radius $2.0,3.0$, and 4.5 virtual units [vu]) and two travel speeds ( 1.5 and 2.0 virtual units/s). Left, The relationship between the homing vector length ( $y$-axis) and the arc length traveled around the loop $(x$-axis) during the trial. The homing vector increased during initial movement, peaked halfway around the loop, then decreased back to zero, with an additional increase again for overshoot trials. The three curves represent the three loop sizes used. Right, The relationship between the homing vector model ( $x$-axis) and the alternative degrees-from-home model ( $y$-axis). The three loop sizes are shown in ascending order of radii. The homing vector length when at the 180 degree point varies substantially between the three radii, at 4, 6, or 9 virtual units away from home.

(Epstein and Vass, 2013; Sherrill et al., 2013), and that the RSC contributes to spatial orientation (Cho and Sharp, 2001; Baumann and Mattingley, 2010; Marchette et al., 2014). We created an anatomical ROI mask of the left and right hemisphere hippocampi and the entire left and right parahippocampal gyri using the Wake Forest University Pick-Atlas automatic anatomical labeling (Maldjian et al., 2003) available for SPM. The Wake Forest University Pick-Atlas does not have an anatomical ROI for the RSC, so we created an ROI using the anatomical tracing program ITK-SNAP (Yushkevich et al., 2006). Our RSC structural ROI was traced along the anatomical boundaries and Brodmann areas outlined by Vann et al. (2009) and Damasio, (2005), including the extreme posterior cingulate and the most ventral and posterior areas of the precuneus, without extending into the occipital-parietal sulcus. We combined the hippocampal, parahippocampal gyrus, and RSC ROIs and resampled to the appropriate image space in SPM. We applied a voxelwise statistical threshold of $p<0.05$ to the contrast maps. To correct for multiple comparisons, we applied a cluster-extent threshold technique. The $3 \mathrm{dCl}$ ustSim program in the AFNI software package was used to conduct a 10,000 iteration, $6 \mathrm{~mm}$ autocorrelation Monte Carlo simulation of the ROI volume (5041 voxels); a minimum voxel extent of 113 was determined to maintain a family-wise error rate of $p<0.05$.

For the whole-brain analysis, a voxelwise statistical threshold of $p<$ 0.01 was applied to the whole-brain contrast maps. Similar to the ROI analysis, 3dClustSim was used to conduct a 10,000 iteration, $6 \mathrm{~mm}$ autocorrelation Monte Carlo simulation analysis on voxels within the group functional brain space using the ResMS header file (167,038 total voxels). From this simulation, a minimum voxel extent of 145 was determined to maintain a family-wise error rate of $p<0.01$. In light of recent suggestions that a more conservative threshold should be used for wholebrain fMRI analyses (Woo et al., 2014), we also applied a more conservative voxelwise threshold of $p<0.001$ with a cluster extent of 35 voxels (cluster corrected to $p<0.05$ ) to our dataset. We used Damasio, (2005) and Pruessner et al. $(2000,2002)$ as references for localization.

Parametric analysis of homing vector. A model-based approach was taken to test the hypothesis that hippocampal, parahippocampal, and retrosplenial activation tracks Euclidean distance from the home location during successful path integration, using a within-subject parametric analysis on the entire length of the loop task. The loops were divided into $1 \mathrm{~s}$ intervals, with a parametric modulator corresponding to each time point along the loop (Spiers and Maguire, 2007; Sherrill et al., 2013) (Fig. 2A). We used stick functions (onsets with zero duration) to sample at each second of each trial, sampling over the entire course of the loop video. The primary parametric modulator was the Euclidean distance to the home location at each time point (chord length in virtual units), which we refer to as a "homing vector." Euclidean distance from the home location varied during the course of the trial: the distance increased 
as the loop moved further from home, cresting halfway around the loop, and then decreased again as the loop returned to the home location (e.g., $1.47,2.73,3.61, \ldots, 1.98,0.56$ ). For overshoot trials, this distance begins to increase again after passing the home location. Figure $2 C$ (left) illustrates the relationship between the homing vector and the total arc length traveled during the trial. Correct and incorrect trials were modeled separately to account for more variance, but only correct trials were examined in our subsequent $t$ test. If a participant was correct on all 36 trials, a total of 431 time points over all the trials would be included in the correct trials parametric modulator.

This model-based approach tests the hypothesis that the hippocampus, RSC, and PHC track Euclidean distance from a home location during path integration; however, it is possible that an alternative model that follows a similar trajectory could provide a better fit for the data. Thus, in addition to the homing vector analysis, we also created an alternative parametric modulator to test a model corresponding to the degrees of arc from the home location at each time point. Critically, by including three loop sizes in our design, this analysis tests for the possibility that brain regions could be responsive to the 180 degree point of the circle (furthest point from home), rather than Euclidean distance from home per se.

Figure $2 B$ illustrates the similarities and differences between the two models for a single loop trial. The degrees-from-home model follows a linear increase and decrease with a maximum at the 180 degree mark because degrees accumulate linearly based on travel speed. In contrast, chord length is related to a sine function, and so the homing vector model arcs. Yet, when looking across the loop sizes, Figure $2 C$ (right) illustrates that, at the 180 degree point, the three loop sizes are quite different in terms of the Euclidean distance from home (homing vector) model, but they are equal in the model using degrees of arc from home. For example, for the 2 unit radius loop, the furthest distance from home is 4 units; however, 4 units away from home on the 3 unit radius loop occurs at $\sim 90$ degrees from home and at only $\sim 55$ degrees from home on the 4.5 unit radius loop. Conversely, the Euclidean distance at 180 degrees from home differs substantially for the three loop sizes, at 4, 6, and 9 units for the 2, 3, and 4.5 unit radii loops, respectively. Accordingly, the regressor for degrees of arc to home was the number of degrees in the arc that the point was from the home location (e.g., 42.97, 85.94, 128.92, ..., 59.21, 16.24). A correlation between the 431 possible time points for all of the trials using the different parameters indicated that degrees of arc from home was correlated with homing vector length $\left(r_{(429)}=0.840, p<\right.$ 0.001 ) because they both broadly increase and decrease at the same rate. Thus, these two alternative models are qualitatively similar and have shared variance; but by contrasting the models, we can determine which model is a better quantitative fit for the data. In contrast with simple localization, the model comparison approach indicates that one model describes the data better than an alternative, providing greater explanatory power behind how a cognitive process is implemented in the brain areas of interest (O'Doherty et al., 2007). This approach has been used to study a variety of cognitive processes in learning, memory, and perception, and provides a means for testing models where shared variance is expected (Ribas-Fernandes et al., 2011; Simon and Daw, 2011; Harvey et al., 2013; Davis et al., 2014; Barendregt et al., 2015).

To compare these two models, we first created separate models in SPM for the Euclidean distance (homing vector) and degrees-from-home parametric modulators. We created separate models, rather than follow the conventional approach of putting both within the same GLM because the GLM approach removes potentially important shared variance, thereby reducing power to detect regions that support either the homing vector or degrees-from-home model. Thus, to effectively compare the models, it is important to include the shared variance in both models. The two models we created included separate regressors for both correct trials and incorrect trials to account for variance attributed to incorrect trials, and also included the motion and signal intensity spike regressors described earlier. The parameter estimates for the parametric modulation for only correct trials from each model were loaded into a onesample $t$ test against 0 to test whether the models significantly described the data. We conducted ROI and whole-brain analyses on both the homing vector and degrees of arc models, using the ROI, voxelwise thresholds, and cluster extents described earlier. For visualization purposes, parameter estimates were extracted from $5 \mathrm{~mm}$ spheres (excluding any voxels with signal dropout) centered on peak coordinates to illustrate the relationship between signal and Euclidean distance from home.

After determining whether the two models significantly described the loop closure data, we next compared the two models. Because the SPMs were created separately, we normalized each parameter estimate $(\beta)$ into a $t$-statistic by dividing them by the SE of the $\beta$ value, to ensure that they were comparable. The SE was computed from the ResMS file (ResMS), the covariance matrix value for that $\beta$ value (Bcov), and the number of time points that were part of the $\beta(\mathrm{N})$ : beta_norm $=\beta /(\operatorname{sqrt}(\operatorname{ResMS} \times \operatorname{Bcov}) / \operatorname{sqrt}(\mathrm{N}))$. We contrasted the $t$-statistics for the different models in SPM8 by conducting a paired $t$ test between the two SPMs.

Analysis of successful navigation. We next examined brain regions that contributed to successful path integration by contrasting BOLD activation in correct trials and incorrect trials, using a boxcar function to model the entire trial duration. The model was analyzed using a standard GLM approach whereby participant-specific parameter estimates pertaining to each regressor were calculated. This model included separate regressors for correct trials and incorrect trials, as well as the motion and signal intensity spike regressors described above. These parameters were convolved with the canonical hemodynamic response function in SPM8. The $t$-contrast between correct trials and incorrect trials was constructed for each participant. Group-averaged statistical parametric maps (SPMs) were created by entering the Correct $>$ Incorrect contrast images from each participant into a one-sample $t$ test using participant as a random factor. We conducted ROI and whole-brain analysis, using the ROI, voxelwise thresholds, and cluster extents described above.

\section{Results}

\section{Behavioral results}

Mean proportion correct in the loop task was $0.588(\mathrm{SD}=0.110)$, which was significantly greater than chance (chance $=0.500$; onesample $t$ test, $t_{(23)}=3.924, p=0.001$ ). Participants were significantly more accurate for trials in which the loop did not end at the home location ("mismatch") than for trials that ended at the home location ("match") (paired $t$ test, $t_{(23)}=-4.485, p<0.001$ ), but were not faster in their responses $\left(t_{(23)}=1.069, p=0.296\right)$. A $3 \times 2$ (loop radius size, speed) repeated-measures ANOVA found that there were no main effects or interactions of movement speed or loop radius of curvature on proportion correct (all $p>0.2$ ), suggesting that participants were not better at one type of loop. An additional $3 \times 3$ (loop radius size, match status) repeated-measures ANOVA found that there was a main effect of match status (overshoot, undershoot, or return to home) on proportion correct $\left(F_{(2,46)}=7.725\right.$, $p=0.001)$, with nonmatches having a greater proportion correct than matches, but no differences in proportion correct between undershoots and overshoots ( $p=0.989$, Bonferroni corrected), in agreement with the paired $t$ tests. There was no main effect of loop size $(p=0.794)$ and no interaction between loop size and match status $(p=0.373)$. Together, these findings indicate that analyses using only correct trials, or those comparing correct trials with incorrect trials, were not disproportionately driven by one speed or one loop size. Performance in the loop task did not correlate with any of the spatial abilities tests (all $p>0.2$ ).

Mean response time was $891.79 \mathrm{~ms}(\mathrm{SD}=116.56)$. There was no difference in reaction time between correct and incorrect trials $\left(t_{(23)}=-0.757, p=0.457\right)$. Participants responded faster to mismatch trials that overshot the home location than for undershoots $\left(t_{(23)}=-7.748, p<0.001\right)$, suggesting that they were aware of the overshoot, but there was no difference in accuracy between overshoot and undershoot trials $\left(t_{(23)}=-0.996, p=\right.$ 0.330). An additional $3 \times 3$ (loop radius size, match status) repeated-measures ANOVA showed no main effect of loop radius size $(p=0.777)$ or interaction $(p=0.143)$. However, the 


\section{Homing Vector Model: Euclidean Distance to Goal}
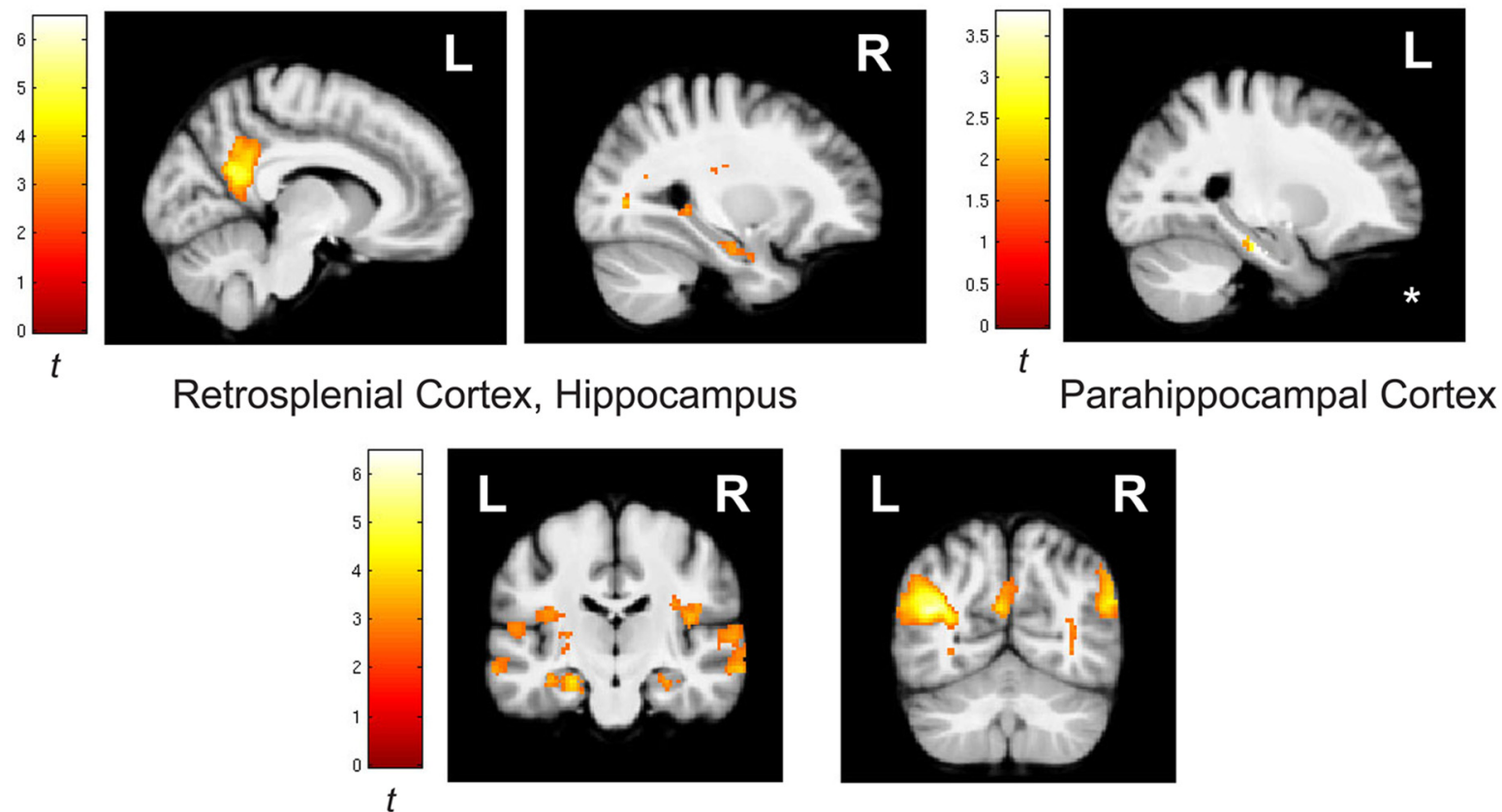

Hippocampus, Angular Gyrus

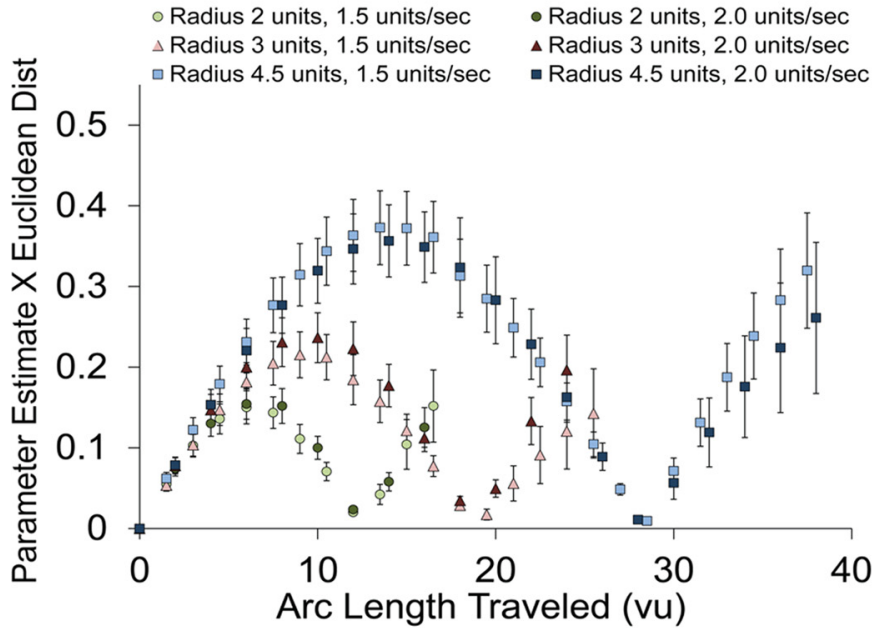

Left Hippocampus

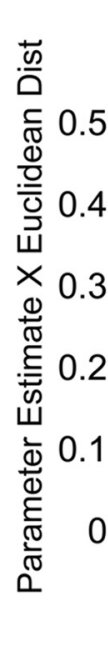

$\square$ Radius 4.5 units, 1.5 units $/ \mathrm{sec}$ $\Delta$ Radius 3 units, 1.5 units/sec - Radius 2 units, 1.5 units/sec

Radius 4.5 units, 2.0 units/sec $\triangle$ Radius 3 units, 2.0 units/sec - Radius 2 units, 2.0 units/sec

Figure 3. Results of the parametric analysis, using the homing vector model. Top, Whole-brain and ROI results of the homing vector model of path integration, indicating brain areas that had an increase in activation with increased Euclidean distance from the home location. Hippocampus and retrosplenial cortex had significant activation at both the Rol level and the whole-brain level, which is shown here. Parahippocampal cortex results were significant at the ROI level only; a masked ROI was used to illustrate these results $\left({ }^{*}\right)$. Whole-brain results are at voxelwise threshold of $p<$ 0.01 , cluster corrected for multiple comparisons to $p<0.01$ (cluster extent 145 voxels). R0I results are at voxelwise threshold of $p<0.05$, cluster corrected to $p<0.05$ (cluster extent 113 voxels). Bottom, To illustrate that signal increased and decreased with Euclidean distance from home, the parameter estimate of the homing vector model is shown relative to the arc length traveled (compare with Fig. 2) for the peak voxel of left hippocampus and left retrosplenial cortex. These figures were derived by multiplying the parameter estimate extracted from $5 \mathrm{~mm}$ spheres (excluding voxels with signal dropout) centered on peak coordinates for the homing vector regressor by the distance from home corresponding to each time point. This illustration is thus predictive based on the parameter estimate, which is derived by the best estimation of the model using those parameters. Mean values were computed from all of the correct trials from all subjects in a given loop radius size/speed condition. Error bars indicate SE, as computed based on the total number of correct trials included in each mean.

ANOVA did find a main effect of match status $\left(F_{(2,46)}=34.721\right.$, $p<0.001$ ), with overshoots (mean $=769.43 \mathrm{~ms}$ ) response time significantly faster than matches (mean $=903.97 \mathrm{~ms} ; p<0.001$, Bonferroni corrected) and undershoots $(p<0.001$, Bonferroni corrected). In addition, response times for match trials were faster than for undershoots (mean $=1000.52 \mathrm{~ms} ; p=0.016$, Bonferroni corrected). This result confirms that participants were prepared to respond for the overshoots and were likely slower for the undershoot trials because they were not prepared for the trial to end early. A $3 \times 2$ (loop radius size, speed) repeated-measures ANOVA found that there were no main effects or interactions of movement speed or loop radius of curvature on reaction time (all $p>0.15$ ), suggesting that participants were not better at one type of loop. 
Table 1. Whole-brain results for the Euclidean distance to the home location, areas showing parametrically increasing activity with increased distance from home ${ }^{a}$

\begin{tabular}{|c|c|c|c|c|}
\hline \multirow[b]{2}{*}{ Brain region } & \multicolumn{2}{|l|}{ Left } & \multicolumn{2}{|l|}{ Right } \\
\hline & $t$ & MNI $x, y, z$ & $t$ & MNI $x, y, z$ \\
\hline \multicolumn{5}{|l|}{ Regions with increasing activity } \\
\hline Inferior frontal gyrus & 3.99 & $-42,34,-4$ & & \\
\hline Sylvian fissure & 3.50 & $-44,-10,14$ & 4.19 & $38,-12,20$ \\
\hline Precentral gyrus & & & 3.44 & $56,-2,8$ \\
\hline Pallidum & 3.04 & $-24,-10,-4$ & & \\
\hline Amygdala & & & 3.28 & $26,0,-24$ \\
\hline Hippocampus (head) & $4.32^{*}$ & $-26,-16,-16$ & 3.37 & $28,-18,-18$ \\
\hline Hippocampus (tail) & 3.92 & $-22,-40,4$ & 3.22 & $30,-38,0$ \\
\hline Retrosplenial cortex & $4.99^{*}$ & $-6,-56,18$ & 3.74 & $10,-54,24$ \\
\hline Temporal pole & 4.06 & $-44,16,-38$ & $4.04^{*}$ & $42,16,-44$ \\
\hline Superior temporal gyrus & 3.44 & $-48,-8,-4$ & 3.89 & $58,-24,8$ \\
\hline Superior temporal sulcus & $4.41^{*}$ & $-58,-36,-2$ & 3.61 & $62,-24,-2$ \\
\hline Middle temporal gyrus & $6.18^{*}$ & $-60,4,-20$ & $6.45^{*}$ & $60,8,-32$ \\
\hline Precuneus & $4.57^{*}$ & $-4,-58,28$ & 3.42 & $6,-58,32$ \\
\hline Angular gyrus & $5.86^{*}$ & $-38,-60,24$ & $4.30^{*}$ & $54,-62,26$ \\
\hline Calcarine sulcus & 3.76 & $-24,-76,4$ & 3.42 & $24,-76,6$ \\
\hline
\end{tabular}

${ }^{a}$ Whole-brain results are at voxelwise threshold of $p<0.01$, cluster corrected for multiple comparisons to $p<0.01$ (cluster extent 145 voxels).

${ }^{*} p<0.001$.

\section{Brain regions coding for a homing vector}

Euclidean distance from the home location varied during the course of each trial: the distance increased as the loop moved further from home, cresting halfway around the loop (corresponding to 180 degrees), and then decreased again as the movement returned home (Fig. $2 A$ ). Because this variable tracks the home location at all times, we refer to it as the "homing vector" (Fujita et al., 1990; Philbeck et al., 2001). The task used three sizes of loop and two movement speeds to provide a variety of homing vector lengths and to dissociate homing vector distance from other factors such as arc length traveled (Fig. 2C, left) and degrees from home (Fig. 2C, right). Here we report the MNI coordinates, cluster size $(k), t$ value, and uncorrected $p$ value for the peak voxel in each significant cluster within our ROI. We used a parametric analysis (see Materials and Methods) to test the hypothesis that activation would increase in this ROI with increasing Euclidean distance from home, that is, increasing length of the homing vector. In line with this prediction, we found significantly greater activation with increasing Euclidean distance from the home location in the following a priori ROI regions: right hippocampus head $\left(x, y, z: 28,-18,-18 ; k=367 ; t_{(23)}=3.37, p=0.0013\right)$, left hippocampus head, body, and tail $(x, y, z:-26,-16,-16 ; k=$ $\left.686 ; t_{(23)}=4.32 ; p=0.0001\right)$, left PHC bordering the subiculum region $\left(x, y, z:-20,-20,-22 ; k=686 ; t_{(23)}=3.28 ; p=0.0016\right)$, and left RSC $\left(x, y, z:-6,-56,18 ; k=273 ; t_{(23)}=4.99 ; p=\right.$ 0.00002 ) (Figure 3 ), supporting our hypothesis that the hippocampus, PHC, and RSC track Euclidean distance to the home location, consistent with the homing vector model.

A whole-brain analysis at a threshold of $p<0.01$ examining areas responsive to increasing Euclidean distance demonstrated activation in bilateral hippocampus and bilateral RSC, as well as bilateral angular gyrus, bilateral precuneus, and bilateral superior temporal gyrus and sulcus (for complete results, see Fig. 3; Table 1). We also examined our results at a more conservative voxelwise threshold of $p<0.001$ (Woo et al., 2014) for the primary analysis of regions showing increasing activity with distance from the home location. Using this more conservative threshold, activity was localized to the left hippocampus and bilateral RSC extending to precuneus (with greater activation on the left), as well as bilateral angular gyrus, bilateral middle temporal gyrus, and right temporal pole.
An alternative explanation is that these areas track the distance from home in terms of degrees of arc. In other words, these areas could be sensitive to being 180 degrees away from the goal, rather than tracking distance per se. To test this hypothesis, we created a model with degrees of arc from the goal as the parametric modulator (for illustrations of the two models, see Fig. $2 B, C$, right). This analysis demonstrated that some of the brain regions that respond to the degrees-from-home model were consistent with those found for the homing vector (Euclidean distance) model: the ROI analysis found activation in bilateral hippocampus and left RSC, and the $p<0.01$ whole-brain level found activation in the left RSC and left hippocampus. Yet, at the more conservative $p<0.001$ level, none of the a priori regions was related to the degrees-from-home model, with activations at this level instead centered on superior temporal gyrus and sulcus, angular gyrus, precentral gyrus, and mPFC.

Contrasting these two models in our a priori ROI showed that bilateral RSC, bilateral hippocampus head, left hippocampal tail, and bilateral PHC (left PHC proper and bilaterally more anteriorly near the border with the subiculum) had greater activation $(p<0.05)$ for the Euclidean distance homing vector model than for the degrees-from-home model, suggesting that these regions are sensitive to Euclidean distance rather than the portion of the loop that has been traversed (Fig. 4). The left PHC $(p<0.001)$ extending into collateral sulcus and bilateral RSC (left $p<0.005$, right $p<0.01)$ results held at the whole-brain level. Additional areas that showed greater consistency with the homing vector model than the degrees-from-home model at the whole brain $(p<0.01)$ included bilateral angular gyrus, left superior parietal lobule, and left lateral occipital gyrus. The opposite contrast (whole brain, $p<0.01$ ) showed that bilateral lingual gyrus, left calcarine sulcus, and bilateral anterior insula/Sylvian fissure were more consistent with the degrees-from-home model than the Euclidean distance homing vector model.

Together, these results suggest that hippocampus, $\mathrm{PHC}$, and RSC are sensitive to Euclidean distance, consistent with the homing vector model. In addition, the results suggest the homing vector model tended to describe the data better in these regions than the alternative degrees-from-home model. It is worth noting that the statistical thresholds for this advantage ranged from $p<0.05$ to $p<0.001$. Figure $2 B, C$ illustrates the differences between these two alternative models, which is elaborated in Materials and Methods. Although the Euclidean distance and degrees-from-home models are broadly correlated because they both increase to the 180 degree mark, the three loop sizes help separate out the effects of the two models. The finding of greater correspondence with Euclidean distance rather than the 180 degree mark suggests that activations in hippocampus, PHC, and RSC are likely tied to Euclidean distance rather than degrees of arc.

\section{Successful path integration}

We contrasted brain activity for correct trials compared with incorrect trials on a within-subject basis to determine brain regions that are involved when successfully performing the loop closure task (Fig. 5). Univariate analysis of the a priori ROI found significantly more left RSC activation $(x, y, z:-2,-44,20 ; k=$ $\left.116 ; t_{(23)}=3.19 ; p=0.002\right)$, right hippocampal activation $(x, y, z$ : $\left.24,-32,-6 ; k=237 ; t_{(23)}=2.19 ; p=0.019\right)$, and right $\mathrm{PHC}$ activation $\left(x, y, z: 20,-30,-18 ; k=237 ; t_{(23)}=2.48 ; p=0.010\right)$ in correct trials than in incorrect trials. Whole-brain analysis at the $p<0.01$ threshold found activation in bilateral RSC, bilateral medial PFC (mPFC), left anterior cingulate, bilateral poster- 


\section{Homing Vector Model > Degrees from Home Model}
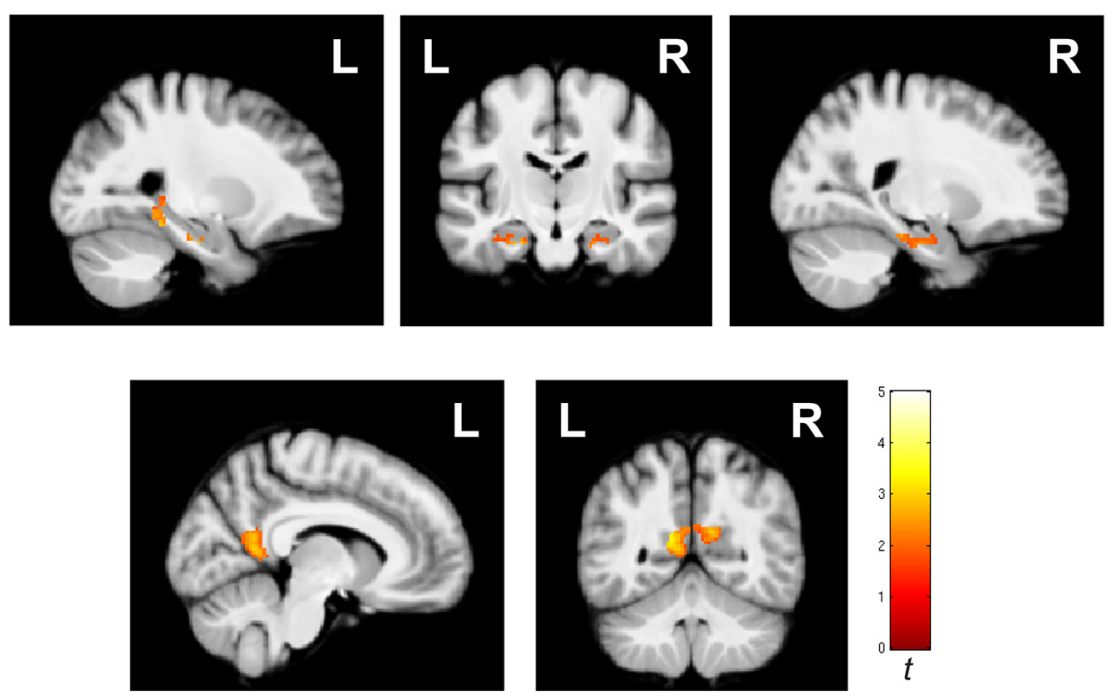

Figure 4. Results for the model comparison contrast of Euclidean distance homing vector model greater than the degrees-fromhome model. A masked ROI was used to illustrate these results. ROI results are at a voxelwise threshold of $p<0.05$, cluster corrected to $p<0.05$; cluster extent 113 voxels.

ior cingulate, bilateral angular gyrus, bilateral fusiform gyrus, and bilateral precuneus. At the more conservative whole-brain threshold of $p<0.001$, we found activation in the left border of posterior cingulate and RSC, bilateral $\mathrm{mPFC}$, bilateral precuneus, left angular gyrus, left middle frontal gyrus, right fusiform gyrus, right calcarine sulcus, and right lateral occipital gyrus. Complete results are listed in Table 2.

\section{Discussion}

Our primary finding is that the hippocampus, RSC, and PHC track Euclidean distance from the home location, supporting a homing vector model of path integration. In addition, the results suggest that a homing vector model tended to describe the data better in these brain regions than an alternative model tracking the degrees from the home location. In addition, we found that the hippocampus, RSC, and PHC, as well as $\mathrm{MPFC}$, were significantly more active in successful path integration trials than in unsuccessful trials. These findings provide insight into the dynamic processes required for path integration in humans.

\section{Hippocampus, RSC, and PHC track Euclidean distance to a home location}

Our results demonstrate a role for the hippocampus in coding Euclidean distance from a home location. In goal-oriented navigational tasks, Sherrill et al. (2013) found increased activation in the posterior hippocampus with increasing proximity to a goal, and Viard et al. (2011) found that anterior hippocampus was more active during test when the goal was closer to the start location. In contrast, Howard et al. (2014) found increasing Euclidean distance from a target (goal) was associated with increased anterior hippocampal activation, but posterior hippocampus activation was associated with increased path length. Likewise, Morgan et al. (2011) found evidence suggesting that anterior hippocampal activity increases with distance. Spiers and Maguire (2007) similarly found increased activation with greater distance from a goal in right subiculum/entorhinal cortex. What these tasks have in common is a design in which the navigator traveled to a known goal location, and in some cases, including

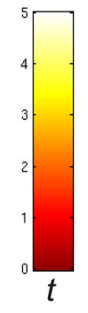

our own previous work (Sherrill et al., 2013), navigators saw an overhead map of the environment before moving, which allows the navigator to anchors to either the initial start point or to the final goal location. In the present experiment, participants traveled an unknown distance along a loop and could only construct a "map" of the loop online during the movement. In this design, there is only one anchor point (the start location), which provides support for the hypothesis that the hippocampus is coding Euclidean distance to the home position. It should be noted that these results are compatible with previous studies suggesting that the hippocampus more generally codes path information, and is also consistent with the idea that it plays a role in tracking distance to a known goal. Finally, although we focus primarily on distance to home here, direction and facing orientation are also important factors for homing behavior (Chadwick et al., 2015).

This study is the one of the first to implicate RSC in online tracking of Euclidean distance. Viard et al. (2011) found increasing RSC activity with proximity to a goal, in contrast to our findings that RSC activity was greatest when furthest from home. Because navigators in our task could only anchor to the home location, task and goal structure could again explain these differences, but together these findings suggest that RSC contributes to an online system that tracks Euclidean distance during path integration. RSC activity has also been observed during navigation in sparse environments (Sherrill et al., 2013), and it is also sensitive to heading direction in both humans and animals (Chen et al., 1994; Marchette et al., 2014). RSC has also been found to support representations of stable landmarks in an environment (Auger et al., 2012; Auger and Maguire, 2013). Although the importance of RSC to spatial navigation has been widely acknowledged, our findings suggest a novel role for RSC as part of the ongoing computation of a homing vector. Finally, we note that the greater sensitivity for the homing vector model than the degrees-from-home model in RSC and hippocampus was found at statistical thresholds of $p<0.01$ and $p<0.05$, respectively, but the results supporting a homing vector model more generally held at the strict $p<0.001$ level for both RSC and the hippocampus.

A third area that tracked Euclidean distance from the home location and was also more active during successful trials than unsuccessful trials was localized to the border between the PHC and subiculum in the ROI analysis. The PHC is known to be involved in landmark navigation (Janzen and van Turennout, 2004; Brown et al., 2010; Epstein and Vass, 2013) and view-based place recognition (Epstein and Kanwisher, 1998). However, the present experiment had no landmarks, and we note that the localization of our PHC results is anterior to and does not overlap with the PPA areas reported in scene and navigation literature (Epstein and Kanwisher, 1998). Although it is possible that some participants attempted to use the arrangement of trees at the home location, this strategy proved difficult due to the large number of trees and quick initial movement away from home. In support of the idea that 


\section{Correct Trials>Incorrect Trials}
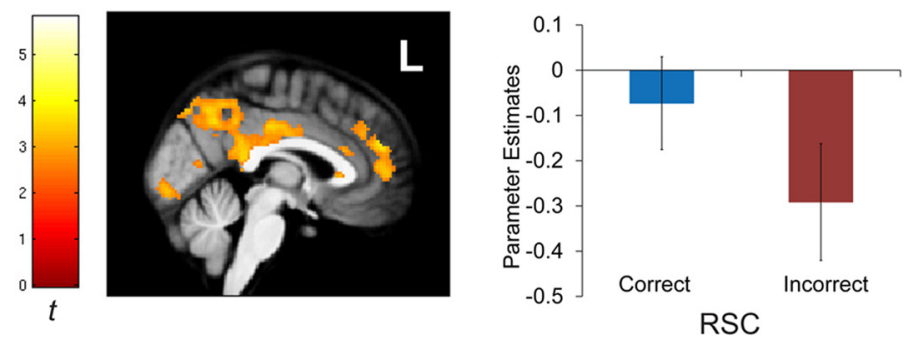

Retrosplenial Cortex, Medial PFC, Cingulate

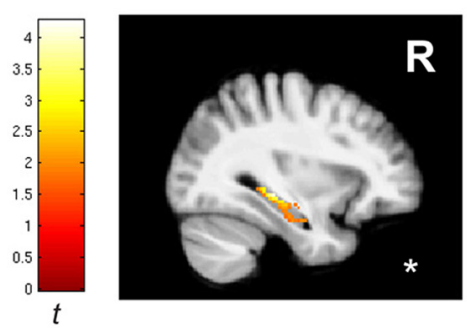

Hippocampus

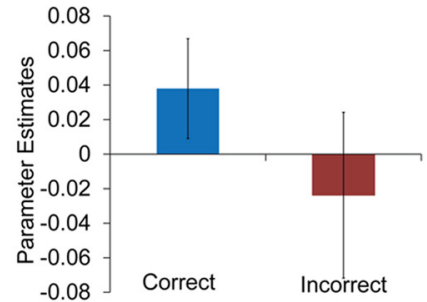

Hippocampus

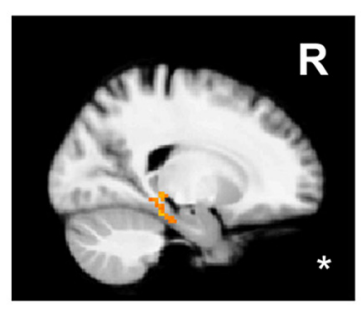

Parahippocampal Cortex

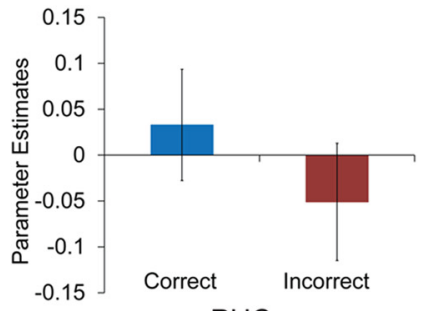

$\mathrm{PHC}$

Figure 5. Results for activity greater for correct trials than incorrect trials. Top, Whole-brain activity for correct trials contrasted with incorrect trials, which included retrosplenial cortex, medial $\mathrm{PFC}$, and cingulate. Bottom, ROI for correct trials contrasted against incorrect trials. Hippocampal and parahippocampal results were significant at the ROI level only; a masked ROI was used to illustrate these results $\left(^{*}\right)$. Whole-brain results are at voxelwise threshold of $p<0.01$, cluster corrected for multiple comparisons to $p<0.01$ (cluster extent 145 voxels). ROI results are at a voxelwise threshold of $p<0.05$, cluster corrected to $p<0.05$ (cluster extent 113 voxels). For illustration purposes, the parameter estimates for the peak voxels were extracted from $5 \mathrm{~mm}$ spheres (excluding voxels with signal dropout) centered on peak coordinates within the ROI are shown. Error bars indicate SEM.

Table 2. Whole-brain results for activations in the loop task for the contrast of correct trials $>$ incorrect trials ${ }^{a}$

\begin{tabular}{|c|c|c|c|c|}
\hline \multirow[b]{2}{*}{ Brain region } & \multicolumn{2}{|l|}{ Left } & \multicolumn{2}{|l|}{ Right } \\
\hline & $t$ & MNI $x, y, z$ & $t$ & MNI $x, y, z$ \\
\hline \multicolumn{5}{|l|}{ Correct $>$ incorrect } \\
\hline Superior frontal gyrus & $3.63^{*}$ & $-2,48,22$ & $4.06^{*}$ & $2,48,24$ \\
\hline Anterior cingulate & 3.64 & $-6,24,20$ & & \\
\hline Middle frontal gyrus & $4.70^{*}$ & $-42,14,36$ & 3.69 & $36,8,44$ \\
\hline Posterior cingulate & 3.59 & $-4,-24,36$ & 3.23 & $2,-12,32$ \\
\hline Superior temporal sulcus & 4.41 & $-64,-36,-6$ & $5.67^{*}$ & $60,-38,-6$ \\
\hline Retrosplenial cortex & $4.45^{*}$ & $-10,-44,22$ & 3.14 & $2,-44,18$ \\
\hline Precuneus & $4.45^{*}$ & $-12,-60,38$ & $3.75^{*}$ & $2,-58,40$ \\
\hline Supramarginal gyrus & 4.52 & $-40,-62,42$ & & \\
\hline Angular gyrus & $5.82^{*}$ & $-48,-56,30$ & 3.42 & $54,-58,32$ \\
\hline Superior parietal lobule & & & 3.64 & $20,-72,42$ \\
\hline Lingual gyrus & 3.91 & $-6,-92,-6$ & 4.53 & $8,-86,-6$ \\
\hline Fusiform gyrus & 4.40 & $-18,-82,-8$ & 4.50 & $10,-74,-12$ \\
\hline Lateral occipital gyrus & & & $4.98^{*}$ & $34,-78,-4$ \\
\hline Calcarine sulcus & 3.58 & $-8,-96,0$ & $4.68^{*}$ & $10,-92,2$ \\
\hline Cuneus & & & 3.77 & $10,-90,14$ \\
\hline
\end{tabular}

${ }^{a}$ Whole-brain results are at voxelwise threshold of $p<0.01$, cluster corrected for multiple comparisons to $p<0.01$ (cluster extent 145 voxels).

${ }^{*} p<0.001$.

the PHC supports path integration, other studies from our laboratory have observed PHC activation during a navigation task in a sparse, landmark-free environment (Sherrill et al., 2013). Based on the results reported here, we suggest that the PHC could combine path integration signals to form a representation of "place," which is used during the approach to home. Given that the peak of this area is on the border of the subiculum, a follow-up high-resolution study would be helpful in defining whether the PHC and subiculum both contribute to path integration.

\section{Regions supporting successful path integration}

Our analysis of successful versus unsuccessful loop closure trials demonstrated differences within the RSC, hippocampus, PHC, and $\mathrm{MPFC}$, and these results are consistent with earlier studies demonstrating activation in these areas during successful path integration in different tasks (Wolbers et al., 2007; Sherrill et al., 2013). Anatomically, it is interesting to note that, although there are strong connections from the anterior hippocampus to the $\mathrm{mPFC}$, most of the hippocampal inputs to the RSC arise from the caudal hippocampus (Aggleton et al., 2012). In addition, reciprocal connections from the $\mathrm{mPFC}$ to the hippocampus are very sparse, with information from the $\mathrm{MPFC}$ reaching the hippocampus via the RSC or nucleus reuniens of the thalamus (Aggleton, 2014; Miller et al., 2014; Ito et al., 2015). In our study, two of these areas, the RSC and mPFC, met the more stringent statistical threshold suggested by Woo et al. (2014), and are discussed here first, followed by a discussion of the role of the hippocampus and $\mathrm{PHC}$ in successful path integration.

Computational models predict that information about the direction and speed of movement stemming from head-direction cells in RSC could update grid cell responses, which subsequently update hippocampal place cells and the representation of position in the environment (Burgess et al., 2007; Hasselmo, 2009; Erdem and Hasselmo, 2012). Such updating of path integration signals can be used to form a metric map of the environment, also known as survey knowledge (Chrastil, 2013). In support of these computational models, RSC has anatomical connections with the hippocampus and PHC (Sugar et al., 2011; Aggleton et al., 2012), as well as PFC (Shibata et al., 2004). The activations in hippocampus, RSC, PHC, and mPFC observed during location tracking in the present experiment, as well as with activations during distance and rotation estimations in a related study (E.R.C. et al., under review), are consistent with these models. The mPFC result 
is consistent with studies suggesting it plays a role in metric navigation (Spiers and Maguire, 2007; Viard et al., 2011; Sherrill et al., 2013; Arnold et al., 2014).

The results from the hippocampus and PHC are consistent with the animal literature on their role in path integration (O'Keefe and Nadel, 1978; Whishaw et al., 1997; Save et al., 2001; Fyhn et al., 2004; McNaughton et al., 2006). Human and animal studies of hippocampal lesions have generated conflicting results, with some studies showing no impairments in path integration with hippocampal lesions (Alyan and McNaughton, 1999; Shrager et al., 2008) and others finding impairments that depended on specific aspects of the lesions (Worsley et al., 2001; Philbeck et al., 2004; Kim et al., 2013; Yamamoto et al., 2014), leaving open the possibility of more general distance and path coding functions for the hippocampus (Johnson and Redish, 2007; Pfeiffer and Foster, 2013). In addition, our analysis at the more stringent level indicates a prominent role for RSC and mPFC in successful encoding, suggesting that these regions could support path integration after hippocampal damage.

Finally, we should note that there are other possible models for path integration, including a configural model, whereby the navigator encodes the entire outbound journey (or current allocentric location) and only computes the return trajectory at the end of the outbound path (Fujita et al., 1993; Loomis et al., 1993). Behavioral evidence suggests that humans are capable of both homing vector and configural strategies (Wiener et al., 2011), and we emphasize that the neural mechanisms of the homing vector described here are not necessarily reflective of conscious strategies, but rather of brain processes for successful path integration. The presence of both homing vector and configural systems could resolve some of the conflicting results from hippocampal lesion studies; patients with hippocampal lesions may not be able to access the homing vector system, which we have demonstrated relies on the hippocampus, but could rely on a configural system to complete certain path integration tasks. Interestingly, rodent models that integrate movement to simulate the neural firing properties of grid cells and place cells based on path information may fall more in line with a movement tracking configural model than with a homing vector model (Gothard et al., 1996; Johnson and Redish, 2007), indicating that the hippocampus may subserve multiple aspects of spatial navigation.

This experiment probed the neural underpinnings of path integration in humans using a novel loop closure task. The results suggest that hippocampus, RSC, and PHC support a homing vector model that tracks the Euclidean distance to the home location. These findings present promising new directions for path integration research in both humans and animals by suggesting that tracking position relative to home is possible via vector coding.

\section{References}

Aggleton J (2014) Looking beyond the hippocampus: old and new neurological targets for understanding memory disorders. Proc Soc Biol 281: 1-9. CrossRef Medline

Aggleton JP, Wright NF, Vann SD, Saunders RC (2012) Medial temporal lobe projections to the retrosplenial cortex of the macaque monkey. Hippocampus 22:1883-1900. CrossRef Medline

Alyan S, McNaughton BL (1999) Hippocampectomized rats are capable of homing by path integration. Behav Neurosci 113:19-31. CrossRef Medline

Andersson JL, Hutton C, Ashburner J, Turner R, Friston K (2001) Modeling geometric deformations in EPI time series. Neuroimage 13:903-919. CrossRef Medline

Arnold AE, Burles F, Bray S, Levy RM, Iaria G (2014) Differential neural network configuration during human path integration. Front Hum Neurosci 8:263. CrossRef Medline

Ashburner J (2007) A fast diffeomorphic image registration algorithm. Neuroimage 38:95-113. CrossRef Medline

Auger SD, Maguire EA (2013) Assessing the mechanism of response in the retrosplenial cortex of good and poor navigators. Cortex 49:2904-2913. CrossRef Medline

Auger SD, Mullally SL, Maguire EA (2012) Retrosplenial cortex codes for permanent landmarks. PLoS One 7:e43620. CrossRef Medline

Barendregt M, Harvey BM, Rokers B, Dumoulin SO (2015) Transformation from a retinal to a cyclopean representation in human visual cortex. Curr Biol 25:1982-1987. CrossRef Medline

Baumann O, Mattingley JB (2010) Medial parietal cortex encodes perceived heading direction in humans. J Neurosci 30:12897-12901. CrossRef Medline

Brown TI, Ross RS, Keller JB, Hasselmo ME, Stern CE (2010) Which way was I going? Contextual retrieval supports the disambiguation of well learned overlapping navigational routes. J Neurosci 30:7414-7422. CrossRef Medline

Brun VH, Leutgeb S, Wu HQ, Schwarcz R, Witter MP, Moser EI, Moser MB (2008) Impaired spatial representation in CA1 after lesion of direct input from entorhinal cortex. Neuron 57:290-302. CrossRef Medline

Burgess N, Barry C, O'Keefe J (2007) An oscillatory interference model of grid cell firing. Hippocampus 17:801-812. CrossRef Medline

Byrne P, Becker S, Burgess N (2007) Remembering the past and imagining the future: a neural model of spatial memory and imagery. Psychol Rev 114:340-375. CrossRef Medline

Chadwick MJ, Jolly AE, Amos DP, Hassabis D, Spiers HJ (2015) A goal direction signal in the human entorhinal/subicular region. Curr Biol 25: 87-92. CrossRef Medline

Chen LL, Lin LH, Green EJ, Barnes CA, McNaughton BL (1994) Headdirection cells in the rat posterior cortex: I. Anatomical distribution and behavioral modulation. Exp Brain Res 101:8-23. CrossRef Medline

Cho J, Sharp PE (2001) Head direction, place, and movement correlates for cells in the rat retrosplenial cortex. Behav Neurosci 115:3-25. CrossRef Medline

Chrastil ER (2013) Neural evidence supports a novel framework for spatial navigation. Psychon Bull Rev 20:208-227. CrossRef Medline

Damasio H (2005) Human brain anatomy in computerized images, Ed 2. Oxford: Oxford UP.

Davis T, Xue G, Love BC, Preston AR, Poldrack RA (2014) Global neural pattern similarity as a common basis for categorization and recognition memory. J Neurosci 34:7472-7484. CrossRef Medline

Durrant-Whyte H, Bailey T (2006) Simultaneous localization and mapping: I. IEEE Robot Autom Mag 13:99-108. CrossRef

Epstein RA, Vass LK (2013) Neural systems for landmark-based wayfinding in humans. Philos Trans R Soc Lond B Biol Sci 369:20120533. CrossRef Medline

Epstein R, Kanwisher N (1998) A cortical representation of the local visual environment. Nature 392:598-601. CrossRef Medline

Erdem UM, Hasselmo M (2012) A goal-directed spatial navigation model using forward trajectory planning based on grid cells. Eur J Neurosci 35:916-931. CrossRef Medline

Fujita N, Loomis JM, Klatzky RL, Golledge RG (1990) A minimal representation for dead-reckoning navigation: updating the homing vector. Geogr Anal 22:326-335.

Fujita N, Klatzky RL, Loomis JM, Golledge RG (1993) The encoding-error model of pathway completion without vision. Geogr Anal 25:295-314.

Fyhn M, Molden S, Witter MP, Moser EI, Moser MB (2004) Spatial representation in the entorhinal cortex. Science 305:1258-1264. CrossRef Medline

Gothard KM, Skaggs WE, McNaughton BL (1996) Dynamics of mismatch correction in the hippocampal ensemble code for space: interaction between path integration and environmental cues. J Neurosci 16:80278040. Medline

Harvey BM, Klein BP, Petridou N, Dumoulin SO (2013) Topographic representation of numerosity in the human parietal cortex. Science 341: 1123-1126. CrossRef Medline

Hasselmo ME (2009) A model of episodic memory: mental time travel along encoded trajectories using grid cells. Neurobiol Learn Mem 92: 559-573. CrossRef Medline

Hegarty M, Richardson AE, Montello DR, Lovelace KL, Subbiah I (2002) 
Development of a self-report measure of environmental spatial ability. Intelligence 30:425-447. CrossRef

Howard LR, Javadi AH, Yu Y, Mill RD, Morrison LC, Knight R, Loftus MM, Staskute L, Spiers HJ (2014) The hippocampus and entorhinal cortex encode the path and Euclidean distances to goals during navigation. Curr Biol 24:1331-1340. CrossRef Medline

Ito HT, Zhang SJ, Witter MP, Moser EI, Moser MB (2015) A prefrontalthalamo-hippocampal circuit for goal-directed spatial navigation. Nature 522:50-55. CrossRef Medline

Janzen G, van Turennout M (2004) Selective neural representation of objects relevant for navigation. Nat Neurosci 7:673-677. CrossRef Medline

Johnson A, Redish AD (2007) Neural ensembles in CA3 transiently encode paths forward of the animal at a decision point. J Neurosci 27:1217612189. CrossRef Medline

Kim S, Sapiurka M, Clark RE, Squire LR (2013) Contrasting effects on path integration after hippocampal damage in humans and rats. Proc Natl Acad Sci U S A 110:4732-4737. CrossRef Medline

Kozhevnikov M, Hegarty M (2001) A dissociation between object manipulation, spatial ability, and spatial orientation ability. Mem Cognit 29:745756. CrossRef Medline

Leonard JJ, Feder HJS (2000) A computationally efficient method for largescale concurrent mapping and localization. In: Robotics research, The Ninth International Symposium (ISRR '99) (Hollerbach J, Koditscheck D, eds), pp 169-176. New York: Springer.

Loomis JM, Klatzky RL, Golledge RG, Cicinelli JG, Pellegrino JW, Fry PA (1993) Nonvisual navigation by blind and sighted: assessment of path integration ability. J Exp Psychol Gen 122:73-91. CrossRef Medline

Maldjian JA, Laurienti PJ, Kraft RA, Burdette JH (2003) An automated method for neuroanatomic and cytoarchitectonic atlas-based interrogation of fMRI data sets. Neuroimage 19:1233-1239. CrossRef Medline

Marchette SA, Vass LK, Ryan J, Epstein RA (2014) Anchoring the neural compass: coding of local spatial reference frames in human medial parietal lobe. Nat Neurosci 17:1598-1606. CrossRef Medline

McNaughton BL, Battaglia FP, Jensen O, Moser EI, Moser MB (2006) Path integration and the neural basis of the 'cognitive map.' Nat Rev Neurosci 7:663-678. CrossRef Medline

Miller AM, Vedder LC, Law LM, Smith DM (2014) Cues, context, and longterm memory: the role of the retrosplenial cortex in spatial cognition. Front Hum Neurosci 8:586. CrossRef Medline

Mittelstaedt H, Mittelstaedt M-L (1982) Homing by path integration. In: Avian navigation (Papi F, Wallraff HG, eds), pp 290-297. Berlin: Springer.

Money J, Alexander D (1966) Turner's syndrome: further demonstration of the presence of specific cognitional deficiencies. J Med Genet 3:47-48. CrossRef Medline

Morgan LK, Macevoy SP, Aguirre GK, Epstein RA (2011) Distances between real-world locations are represented in the human hippocampus. J Neurosci 31:1238-1245. CrossRef Medline

O’Doherty JP, Hampton A, Kim H (2007) Model-based fMRI and its application to reward learning and decision making. Ann N Y Acad Sci 1104: 35-53. CrossRef Medline

O’Keefe J, Nadel L (1978) The hippocampus as a cognitive map. Oxford: Clarendon.

Pfeiffer BE, Foster DJ (2013) Hippocampal place-cell sequences depict future paths to remembered goals. Nature 497:74-79. CrossRef Medline

Philbeck JW, Klatzky RL, Behrmann M, Loomis JM, Goodridge J (2001) Active control of locomotion facilitates nonvisual navigation. J Exp Psychol Hum Percept Perform 27:141-153. CrossRef Medline

Philbeck JW, Behrmann M, Levy L, Potolicchio SJ, Caputy AJ (2004) Path integration deficits during linear locomotion after human medial temporal lobectomy. J Cogn Neurosci 16:510-520. CrossRef Medline

Pruessner JC, Li LM, Serles W, Pruessner M, Collins DL, Kabani N, Lupien S, Evans AC (2000) Volumetry of hippocampus and amygdala with high- resolution MRI and three-dimensional analysis software: minimizing the discrepancies between laboratories. Cereb Cortex 10:433-442. CrossRef Medline

Pruessner JC, Köhler S, Crane J, Lord C, Byrne A, Kabani N, Collins DL, Evans AC (2002) Volumetry of temporopolar, perirhinal, entorhinal, and parahippocampal cortex from high-resolution MR images: considering the variability of the collateral sulcus. Cereb Cortex 12:1342-1353. CrossRef Medline

Ribas-Fernandes JJ, Solway A, Diuk C, McGuire JT, Barto AG, Niv Y, Botvinick MM (2011) A neural signature of hierarchical reinforcement learning. Neuron 71:370-379. CrossRef Medline

Save E, Guazzelli A, Poucet B (2001) Dissociation of the effects of bilateral lesions of the dorsal hippocampus and parietal cortex on path integration in the rat. Behav Neurosci 115:1212-1223. CrossRef Medline

Sherrill KR, Erdem UM, Ross RS, Brown TI, Hasselmo ME, Stern CE (2013) Hippocampus and retrosplenial cortex combine path integration signals for successful navigation. J Neurosci 33:19304-19313. CrossRef Medline

Shibata H, Kondo S, Naito J (2004) Organization of retrosplenial cortical projections to the anterior cingulate, motor, and prefrontal cortices in the rat. Neurosci Res 49:1-11. CrossRef Medline

Shrager Y, Kirwan CB, Squire LR (2008) Neural basis of the cognitive map: path integration does not require hippocampus or entorhinal cortex. Proc Natl Acad Sci U S A 105:12034-12038. CrossRef Medline

Simon DA, Daw ND (2011) Neural correlates of forward planning in a spatial decision task in humans. J Neurosci 31:5526-5539. CrossRef Medline

Spiers HJ, Maguire EA (2007) A navigational guidance system in the human brain. Hippocampus 17:618-626. CrossRef Medline

Sugar J, Witter MP, van Strien NM, Cappaert NL (2011) The retrosplenial cortex: intrinsic connectivity and connections with the (para)hippocampal region in the rat: an interactive connectome. Front Neuroinform 5:7. CrossRef Medline

Taube JS, Muller RU, Ranck JB Jr (1990) Head-direction cells recorded from the postsubiculum in freely moving rats: II. Effects of environmental manipulations. J Neurosci 10:436-447. Medline

Vann SD, Aggleton JP, Maguire EA (2009) What does the retrosplenial cortex do? Nat Rev Neurosci 10:792-802. CrossRef Medline

Viard A, Doeller CF, Hartley T, Bird CM, Burgess N (2011) Anterior hippocampus and goal-directed spatial decision making. J Neurosci 31:46134621. CrossRef Medline

Whishaw IQ, McKenna JE, Maaswinkel H (1997) Hippocampal lesions and path integration. Curr Opin Neurobiol 7:228-234. CrossRef Medline

Wiener JM, Berthoz A, Wolbers T (2011) Dissociable cognitive mechanisms underlying human path integration. Exp Brain Res 208:61-71. CrossRef Medline

Wolbers T, Wiener JM, Mallot HA, Büchel C (2007) Differential recruitment of the hippocampus, medial prefrontal cortex, and the human motion complex during path integration in humans. J Neurosci 27:9408 9416. CrossRef Medline

Woo CW, Krishnan A, Wager TD (2014) Cluster-extent based thresholding in fMRI analyses: pitfalls and recommendations. Neuroimage 91:412419. CrossRef Medline

Worsley CL, Recce M, Spiers HJ, Marley J, Polkey CE, Morris RG (2001) Path integration following temporal lobectomy in humans. Neuropsychologia 39:452-464. CrossRef Medline

Yamamoto N, Philbeck JW, Woods AJ, Gajewski DA, Arthur JC, Potolicchio SJ Jr, Levy L, Caputy AJ (2014) Medial temporal lobe roles in human path integration. PLoS One 9:e96583. CrossRef Medline

Yushkevich PA, Piven J, Hazlett HC, Smith RG, Ho S, Gee JC, Gerig G (2006) User-guided 3D active contour segmentation of anatomical structures: significantly improved efficiency and reliability. Neuroimage 31:11161128. CrossRef Medline

Zacks JM, Mires J, Tversky B, Hazeltine E (2000) Mental spatial transformations of objects and perspective. Spat Cogn Comput 2:315-332. 